\title{
Exactly Solvable Chaotic Circuit
}

\author{
Ned J. Corron, Mark T. Stahl, and Jonathan N. Blakely \\ U. S. Army Research, Development and Engineering Command \\ RDMR-WSS, Redstone Arsenal, Alabama 35898 USA \\ ned.corron@us.army.mil
}

\begin{abstract}
We report the construction and operation of a novel chaotic electronic oscillator for which a detailed model admits an exact analytic solution. The circuit is modeled by a hybrid dynamical system including both a differential equation and discrete switching condition. The analytic solution is written as the linear convolution of a symbol sequence and a fixed basis pulse, similar to that of conventional communications waveforms. Waveform returns sampled at the switching times are shown to be conjugate to a chaotic shift map, effectively proving the existence of chaos in the circuit. We show the analytic solution can be used to accurately reconstruct a measured chaotic waveform, thereby confirming the efficacy of the exactly solvable circuit model.
\end{abstract}

\section{INTRODUCTION}

It is commonly assumed that the complexity of chaos precludes straightforward analytic solution. However, this is not necessarily true [1-2]. For difference equations, it has been explicitly shown that trajectories of the shift map and baker's map can be written as the convolution of a random process and an acausal basis pulse [3]. A similar construction is available for analogous continuous-time waveforms [4-6]. Yet in some sense, these toy systems are of limited practical interest, since they are not an accurate model for any observed physical system.

In this paper, we report the construction and operation of a novel chaotic electronic oscillator circuit for which a detailed model admits an exact analytic solution. The oscillator is a hybrid circuit, in that it contains both analog and digital components. A mathematical model of the circuit contains a second order differential equation coupled to a switching condition that controls a binary state. Importantly, the model admits an exact analytic solution, which is written as a linear convolution of binary logic symbols and a fixed basis pulse. Successive returns in the analog state at the discrete transition times are shown to exactly satisfy a shift map, thereby proving the oscillator is chaotic.

In operation, the electronic circuit generates waveforms that closely agree with the analytic solution. Sampled at switching times, the circuit generates a shift map consistent with the analytic model. Using symbols extracted from a measured waveform, we construct the corresponding analytic solution and find it closely matches the observed waveform. Together, these observations confirm that the exactly solvable analytic model provides a good representation of the electronic circuit.

\section{ELECTRONIC CIRCUIT}

A hybrid electronic oscillator, containing both analog and digital components, is shown in Fig. 1. This circuit was constructed using commercially available, discrete components on a solderless breadboard. The analog operational amplifiers are all type TL082, which are powered using $\pm 15 \mathrm{~V}$. The diodes are all type 1N4148. The digital integrated circuit is a dual positive-edge-triggered D flip flop (SN54LS74AJ), which is powered with $+5 \mathrm{~V}$. The digital and analog components share a common ground.

On the left side of the circuit, certain analog components are grouped by dashed boxes. The first grouping, labeled $-R$, comprises an active circuit realizing a negative resistor. The second grouping, labeled $L$, is an impedance converter that provides a nearly ideal inductor. Also significant is the capacitor labeled $C$, which connects $-R$ and $L$ to a virtual ground provided by an operational amplifier. For the nominal circuit values shown in the figure, we have $C=1.3 \mu \mathrm{F}$, $L=2.7 \mathrm{H}$, and $-R$ is tunable by a variable resistor.

We recognize the left side of this circuit as a standard RLC harmonic oscillator, except that the resistance is negative. Thus, this part of the circuit is modeled by the equations

$$
C \frac{d v}{d t}-\frac{v}{R}+i=0
$$

and

$$
L \frac{d i}{d t}=v-v_{s}
$$

where $v$ is the tank voltage, $i$ is the current through the inductor, and $v_{s}$ is a feedback voltage applied to the inductor. We introduce the dimensionless time 


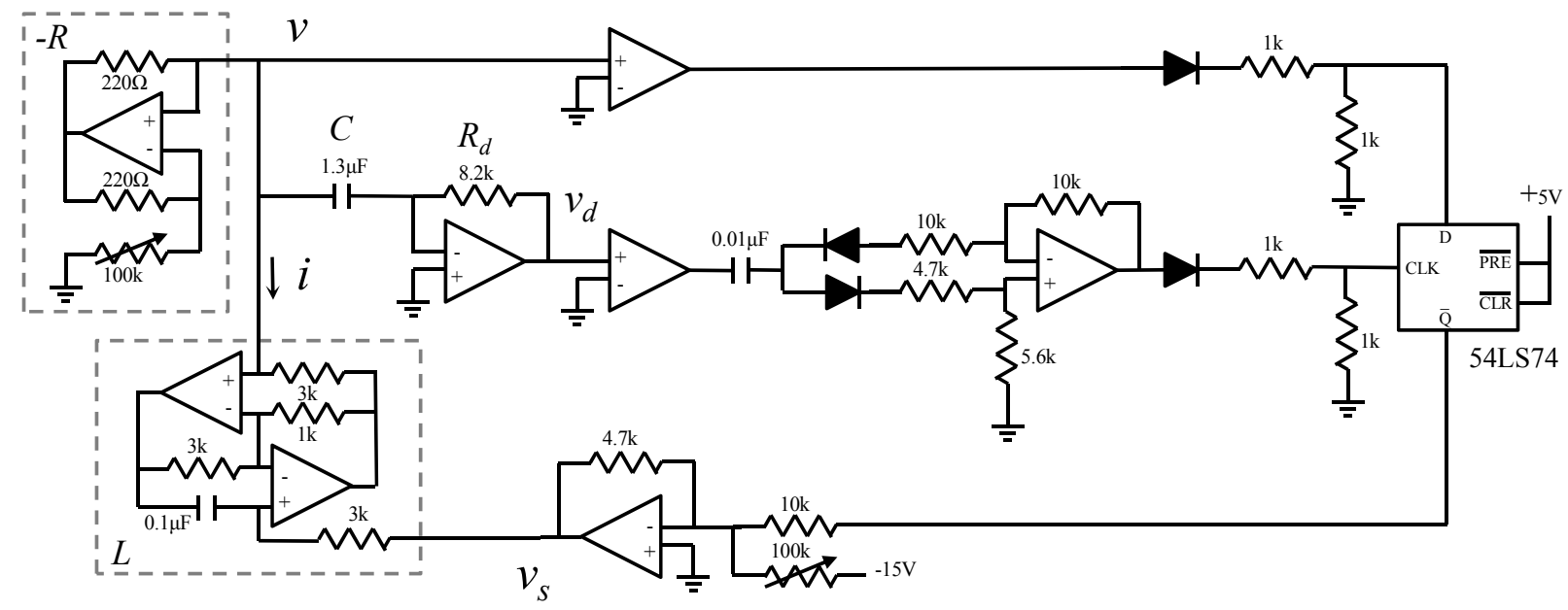

Figure 1. Chaotic hybrid oscillator circuit.

$$
\tau=\frac{t}{T}
$$

where

$$
T=2 R C \omega \sqrt{\frac{L}{4 R^{2} C-L}}
$$

and $\omega=2 \pi$. As we show below, the period $T$ is the return time for the oscillator. Equations (1) and (2) are then written as

$$
\frac{d^{2} v}{d \tau^{2}}-2 \beta \frac{d v}{d \tau}+\left(\omega^{2}+\beta^{2}\right)\left(v-v_{s}\right)=0
$$

where the parameter

$$
\beta=\frac{T}{2 R C}
$$

is the dimensionless negative damping. For the circuit, we only consider $0<\beta \leq \ln 2$.

We now examine the function of the right side of the circuit, which contains the digital circuitry. In the top trace, an operational amplifier is configured as a comparator, which detects the sign of the tank voltage $v$. The subsequent diode and voltage divider convert the saturated amplifier output to digital logic levels. The middle trace uses a current-to-voltage converter to give

$$
v_{d}=-R_{d} C \frac{d v}{d t}
$$

where $R_{d}$ is the feedback resistor. A second comparator then detects the sign of this voltage. The following capacitor, diodes, and difference amplifier generate a short trigger pulse for any transition in the comparator output. Thus, the middle trace generates a trigger pulse whenever the derivative of the tank voltage changes sign. This trigger signal is also scaled to digital logic levels.

The digital logic signals from the top two traces control a single flip flop in the 54LS74 integrated circuit. The flip flop is configured here so that its output encodes and holds the sign of the tank voltage at the last transition in the capacitor current. This output is fed back to the oscillator via the bottom trace. A summing circuit with fixed gain shifts the digital signal to the symmetric levels $\pm V$, and the feedback signal $v_{s}$ is applied to the tank inductor.

Therefore, the feedback circuit is modeled by the switching condition

$$
\frac{d v}{d t}=0 \Rightarrow v_{s}=V \operatorname{sgn}(v)+V_{0}
$$

meaning that, whenever the derivative of the tank voltage passes through zero, the feedback voltage $v_{s}$ is set to the sign of the tank voltage times the fixed magnitude $V$. Furthermore, the feedback voltage is held constant until the next trigger event when the derivative transitions. An offset voltage $V_{0}$ is included to account for a small, yet unavoidable asymmetry in the electronic circuit.

\section{ANALYTIC SOLUTION}

The dynamics of the electronic circuit are modeled by the ordinary differential equation (5) with the switching equation (8). Defining the dimensionless states

$$
u=\frac{v-V_{0}}{V}
$$

and

$$
s=\frac{v_{s}-V_{0}}{V}
$$

we obtain the dimensionless hybrid system 


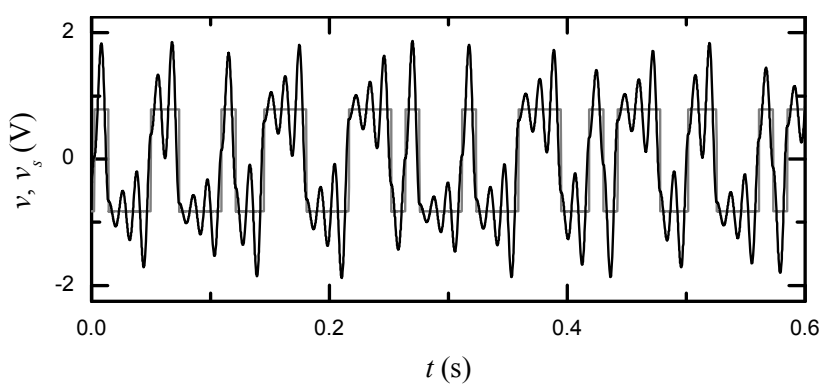

Figure 2. Typical time series measured from the circuit. The oscillating waveform is the tank voltage $v$, while the random square wave is the discrete switching state $v_{s}$.

$$
\ddot{u}-2 \beta \dot{u}+\left(\omega^{2}+\beta^{2}\right) \cdot(u-s)=0
$$

and

$$
\dot{u}(\tau)=0 \Rightarrow s(\tau)=\operatorname{sgn}(u(\tau))
$$

where $\omega=2 \pi$ and a dot represents a derivative with respect to the dimensionless time $\tau$.

Significantly, an exact analytic solution to the hybrid system can be found [6]. Here we write the solution as the linear superposition

$$
u(\tau)=\sum_{m=[\tau]}^{\infty} s_{m} \cdot P(\tau-m)
$$

where the square brackets indicate the largest integer less than or equal to the argument,

$$
P(\tau)=\left\{\begin{array}{cc}
\left(1-e^{-\beta}\right) e^{\beta \tau}\left(\cos \omega \tau-\frac{\beta}{\omega} \sin \omega \tau\right), & \tau<0 \\
1-e^{\beta(\tau-1)\left(\cos \omega \tau-\frac{\beta}{\omega} \sin \omega \tau\right),} & 0 \leq \tau<1 \\
0, & 1 \leq \tau
\end{array}\right.
$$

is a basis pulse, and $s_{m}$ is sequence of binary symbols, \pm 1 . The veracity of this solution can be confirmed by direct substitution.

We note that the solution (13) satisfies $\dot{u}(n)=0$ and $\dot{u}(n+1 / 2)=0$ for all integer $n$. We also note that $\operatorname{sgn}\{u(n+1 / 2)\}=\operatorname{sgn}\{u(n)\}$ for all $n$; thus, transitions in the discrete state $s(\tau)$ due to the switching condition (12) occur only at integer dimensionless times. Thus, we can condense the binary waveform $s(\tau)$ to the discrete sequence of binary symbols, $s_{n}=s(n)$.

Significantly, successive returns of the solution at integer dimensionless times satisfy the recursion relation

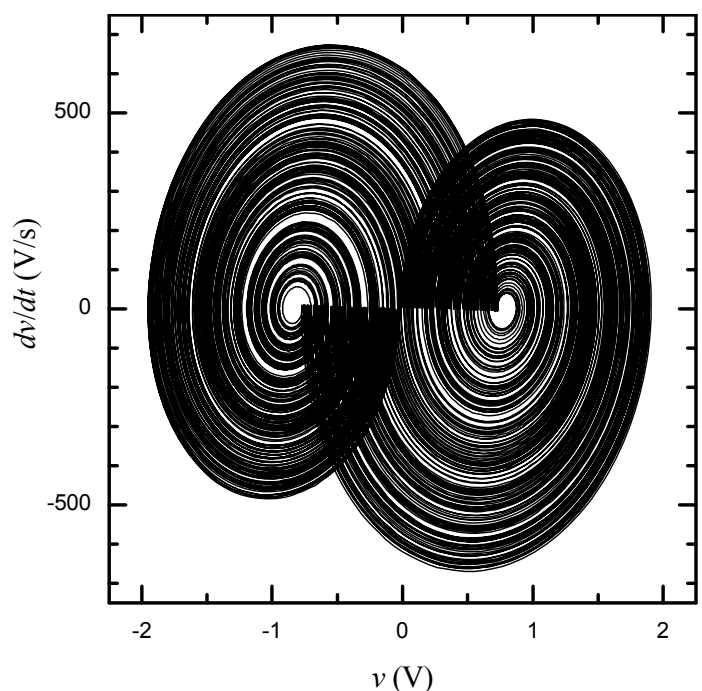

Figure 3. Phase space projection of measured circuit waveforms.

$$
u(n+1)=e^{\beta} u(n)-\left(e^{\beta}-1\right) \cdot s_{n}
$$

which is a shift map with slope $e^{\beta}$. Since $\beta>0$, the slope of the return map is everywhere greater than one. The map is also closed on the unit interval for $\beta \leq \ln 2$, so the shift map is necessarily chaotic. By implication, the hybrid system (11)-(12) is also chaotic, and it is characterized by the positive Lyapunov exponent $\lambda=\beta$.

\section{MEASURED WAVEFORM DATA}

The negative resistor was adjusted to obtain chaotic dynamics. For $\mathrm{R} \sim 6.5 \mathrm{k} \Omega$, the circuit oscillates chaotically with a fundamental frequency near $84 \mathrm{~Hz}$. Waveforms $v, v_{d}$, and $v_{s}$ were sampled at $100 \mathrm{kHz}$ using a data acquisition device and a PC. To reduce sampling noise, the oversampled data was smoothed using a running average over a window of ten samples.

Fig. 2 shows a typical measured time series for the tank voltage $v$. Also shown is the corresponding digital signal $v_{s}$, which switches between two fixed points, $\pm V$. After each switching event, the tank voltage exhibits growing oscillations about the fixed point. When the oscillation gets large, a switching event is triggered. Fig. 3 shows a phase space projection of $v$ and $v_{d}$ for a four-second trajectory. From analysis of the measured signal $v_{s}$, we estimate the amplitude scale $V=0.81 \mathrm{~V}$ and offset $V_{0}=-0.02 \mathrm{~V}$.

Using the nominal circuit values and equations (4) and (6), we estimate $T=0.012 \mathrm{~s}$ and $\beta \doteq 0.7 \sim \ln 2$. However, more precise estimates for $T$ and $\beta$ are derived from analysis of the measured waveform. Using transitions detected in $v_{s}$, we determine the return times $t_{n}$ where $\frac{d v}{d t}\left(t_{n}\right)=0$ and $\left|v\left(t_{n}\right)-V_{0}\right|<V$. The average return time is $T=\left\langle t_{n+1}-t_{n}\right\rangle$, which gives $\mathrm{T}=0.0119 \mathrm{~s}$. A return map for the successive 


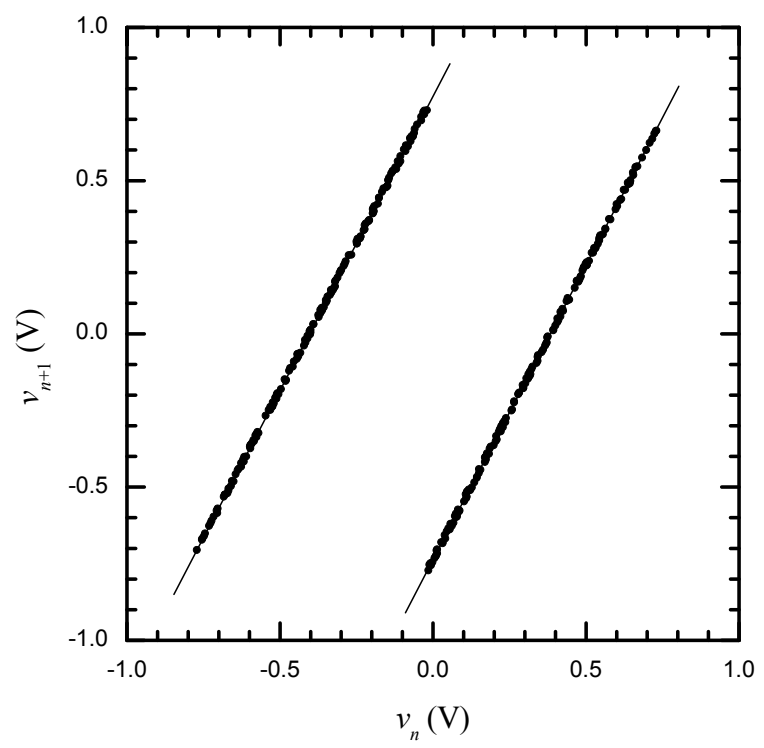

Figure 4. Successive return map with lines fit to measured data. The slope of both lines are $e^{\beta}=1.92$, so that the negative damping is $\beta=0.65$.

returns $v\left(t_{n}\right)$ is shown in Fig. 4. This map is in agreement with the analytic solution (15), which predicts the slope $e^{\beta}>1$. Using a linear least squares fit to each segment of the measured return map, we obtain $e^{\beta}=1.92$, so that $\beta=0.65$.

We also directly confirm the agreement between the oscillator circuit and the analytic model. From the measured switching signal $v_{s}$, we extract the symbol sequence $s_{n}$. For the 4-second waveform, a total of 336 symbols are obtained. Using these symbols and the precise estimates of $T$ and $\beta$, the corresponding analytic waveform is constructed using equations (9) and (13). We note the sum in the analytic model is evaluated for just the known symbols, so that the solution is not theoretically exact; however, the exponential nature of the neglected terms implies the error in the truncated solution is negligible. A typical portion of the measured waveform and analytic solution are shown in Fig. 5(a). In this plot, the two waveforms overlap and are indistinguishable, which indicates remarkable agreement. In Fig. 5(b), the difference $\Delta v$ between the measured waveform and analytic solution is shown on a finer scale. The close agreement between the two waveforms confirms the reliability of the circuit model.

\section{CONCLUSIONS}

In this paper we showed a chaotic oscillator circuit that is accurately modeled by an exactly solvable hybrid system. Although the circuit oscillates at low frequencies $(\sim 84 \mathrm{~Hz})$, a higher frequency version of this oscillator may prove useful for technological applications of chaos. For example, the circuit provides an antipodal chaotic waveform, which is preferable for proposed chaotic communication approaches
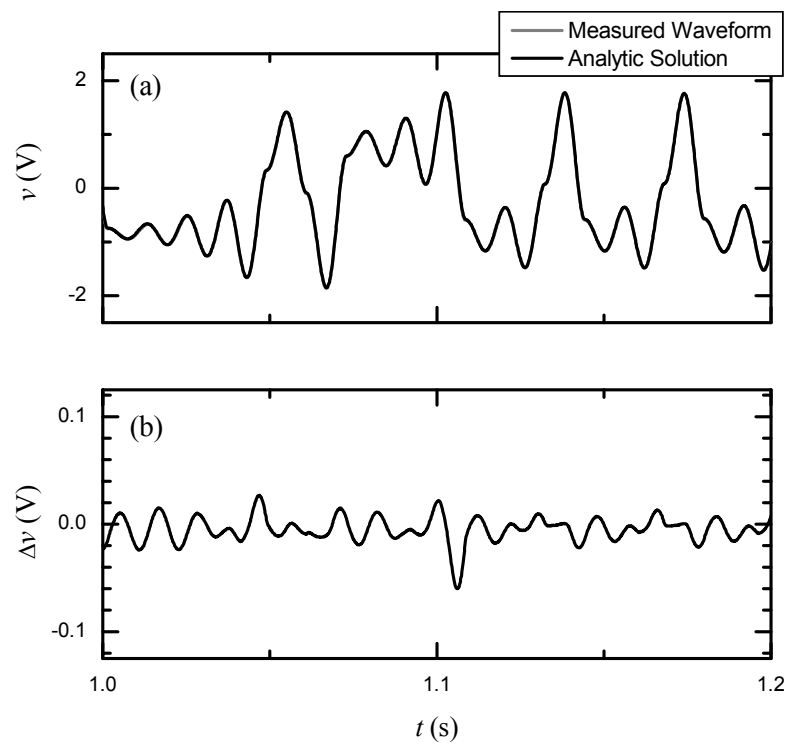

Figure 5. A typical time series comparison showing (a) measured circuit waveform and corresponding analytic solution and (b) magnified

(20x) difference $\Delta v$ between measured and analytic waveforms.

based on encoding message signals in symbolic dynamics [7, 8]. In addition, the existence of a fixed basis pulse suggests that a matched filter may be practically realized for detecting and decoding the received symbols. Along these lines, we have recently identified a significant relationship to the synthesis of reverse-time chaos with linear filters [9]. The development and application of a practical matched filter for chaotic oscillators is the subject of our current research efforts.

\section{REFERENCES}

[1] S. Katsura and W. Fukuda, "Exactly solvable models showing chaotic behavior," Physica A, vol. 130, 597-605, April 1985.

[2] K. Umeno, "Method of constructing exactly solvable chaos," Phys. Rev. E, vol. 55, pp. 5280-5284, May 1997.

[3] D. F. Drake and D. B. Williams, "Linear, random representations of chaos," IEEE Trans. Signal Process., vol. 55, pp. 1379-1389, April 2007.

[4] S. T. Hayes, "Chaos from linear systems: implications for communicating with chaos, and the nature of determinism and randomness," J. Phys. Conf. Series, vol. 23, pp. 215-237, October 2005.

[5] Y. Hirata and K. Judd, "Constructing dynamical systems with specified symbolic dynamics," Chaos, vol. 15, art. 033102, September 2005.

[6] N. J. Corron, "An exactly solvable chaotic differential equation," Dyn. Contin. Discrete Impuls. Syst. A, vol. 16, pp. 777-788, November 2009.

[7] S. Hayes, C. Grebogi, and E. Ott, "Communicating with chaos," Phys. Rev. Lett., vol. 70, pp. 3031-3034, 17 May 1993.

[8] E. M. Bollt, "Review of chaos communication by feedback control of symbolic dynamics," Int. J. Bifurcations Chaos, vol. 13, pp. 269-285, February 2003.

[9] N. J. Corron, S. T. Hayes, S. D. Pethel, and J. N. Blakely, "Chaos without nonlinear dynamics," Phys. Rev. Lett., vol. 97, art. 024101, 14 July 2006. 